\title{
ESSENTIAL IDEALS OF INCIDENCE ALGEBRAS
}

\author{
EUGENE SPIEGEL
}

(Received 19 August 1998; revised 17 August 1999)

Communicated by C. F. Miller

\begin{abstract}
It is determined when there exists a minimal essential ideal, or minimal essential left ideal, in the incidence algebra of a locally finite partially ordered set defined over a commutative ring. When such an ideal exists, it is described.
\end{abstract}

1991 Mathematics subject classification (Amer. Math. Soc.): primary 16D15, 16D25.

In [2], Green and Van Wyk consider the existence of a minimal essential ideal of a structural matrix ring, and question when this ideal is the same as the BrownMcCoy radical of the ring. A structural matrix ring is the incidence algebra of a finite preordered set. In this note we describe the minimal essential ideal and minimal essential left ideal of the incidence algebra, $I(X, R)$, when $X$ is a locally finite partially ordered set and $R$ a commutative ring with identity. Recall that $I(X, R)$ is the set of all functions $f: X \times X \rightarrow R$ with $f(x, y)=0$ unless $x \leq y$, together with the operations

$$
\begin{aligned}
(f+g)(x, y) & =f(x, y)+g(x, y), \\
f g(x, y) & =\sum_{x \leq z \leq y} f(x, z) g(z, y), \\
(r f)(x, y) & =r f(x, y)
\end{aligned}
$$

for

$$
f, g \in I(X, R), \quad r \in R, \quad x, y \in X
$$

(C) 2000 Australian Mathematical Society $0263-6115 / 2000 \$ A 2.00+0.00$ 
If $x, y \in X$, with $x \leq y$, let $e_{x y}$ denote the element of $I(X, Y)$ given by

$$
e_{x y}(u, v)= \begin{cases}1 & \text { if } x=u \text { and } y=v ; \\ 0 & \text { otherwise. }\end{cases}
$$

An ideal, $A$, of a ring $T$, is called essential, or large, if $A \cap B \neq\{0\}$ for any ideal $B \neq\{0\}$. Similarly, the left ideal $A$ is an essential left ideal if $A \cap B \neq\{0\}$ for any non-zero left ideal $B$. Of course, $T$ is essential in $T$, the intersection of two essential ideals is essential, and any ideal containing an essential ideal is essential. Similar statements hold for essential left ideals.

Suppose, now, that $X$ is a locally finite partially ordered set and $R$ a commutative ring with identity. Associate to $X$ the partially ordered set, $I(X)$, ordered by inclusion, of all non-empty intervals, $[x, y]$, with $x, y \in X$. Further, let $\operatorname{Ess}(R)$ be the partially ordered set, ordered by inclusion, of all essential ideals of $R$. If $\operatorname{Max}(I(X))$ is the collection of all maximal elements of $I(X)$, call a function $\phi: \operatorname{Max}(I(X)) \rightarrow \operatorname{Ess}(R)$, an essential function. Suppose $\phi$ is an essential function and let

$$
\begin{aligned}
A_{\phi}= & \{f \in I(X, R) \mid f(x, y) \in \phi([x, y]) \\
& \text { if }[x, y] \in \operatorname{Max}(I(X)), f(x, y)=0 \text { otherwise }\} .
\end{aligned}
$$

It is straightforward to verify that $A_{\phi}$ is an ideal. Notice that when $[x, y] \in \operatorname{Max}(I(X))$, and $K$ is an ideal of $I(X, R)$, then $e_{x x} K e_{y y}$ is an ideal of $I(X, R)$, namely,

$$
e_{x x} K e_{y y}=\left\{f(x, y) e_{x y} \mid f \in K\right\}
$$

This follows by the maximality of $[x, y]$. Indeed, $g e_{x y}=g(x, x) e_{x y}$ and $e_{x y} g=$ $g(y, y) e_{x y}$, for any $g \in I(X, R)$.

We now note some additional ideals of $I(X, R)$. Let $n$ be a positive integer and

$$
Z_{n}(X, R)=\{f \in I(X, R) \mid f(x, y)=0 \text { if }|[x, y]| \leq n\} .
$$

Again it is easy to verify that $Z_{n}(X, R)$ is an ideal of $I(X, R)$. The following lemma shows that the ideals that we have defined give rise to essential ideals.

LEMMA 1. Suppose $X$ is a locally finite partially ordered set and $R$ a commutative ring with identity. Let $n$ be a positive integer and $\phi$ an essential function. Then $A_{\phi}+Z_{n}(X, R)$ is an essential ideal of $I(X, R)$.

Proof. Let $J=A_{\phi}+Z_{n}(X, R)$. Certainly $J$ is an ideal. We check that it is essential. Let $K$ be a non-zero ideal of $I(X, R)$. Suppose that $0 \neq f \in K$ and $f(x, y) \neq 0$. If $[x, y]$ is contained in a maximal interval $[u, v]$, then $u \leq x \leq y \leq v$ and $w=e_{u x} f e_{y v}=f(x, y) e_{u v} \in K$. In particular, $e_{u u} K e_{v v}=\left\{g(u, v) e_{u v} \mid g \in K\right\}$, 
which is contained in $K$, is a non-zero ideal of $I(X, R)$. Let $C=\{g(u, v) \mid g \in K\}$ and $D=\left\{h(u, v) \mid h \in A_{\phi}\right\}$. As $C$ is a non-zero ideal of $R$, and $D=\phi([u, v])$ is an essential ideal of $R$, we have $C \cap D$ is a non-zero ideal of $R$. Then there is a $g \in K$, and an $h \in A_{\phi}$, with $g(u, v)=h(u, v) \neq 0$. Hence $e_{u u} g e_{v v}=e_{u u} h e_{v v} \in K \cap J$. We have thus shown that $J \cap K \neq\{0\}$ when $[x, y]$ is contained in a maximal interval. Suppose, now, that $[x, y]$ is not contained in a maximal interval. Then there is a sequence of intervals

$$
[x, y]=\left[x_{0}, y_{0}\right] \subset\left[x_{1}, y_{1}\right] \subset\left[x_{2}, y_{2}\right] \subset \cdots,
$$

with $\left[x_{i}, y_{i}\right]$ a proper subset of $\left[x_{i+1}, y_{i+1}\right]$, for $i=1,2, \ldots$. Further,

$$
\left|\left[x_{n+1}, y_{n+1}\right]\right| \geq n+1
$$

and $f(x, y) e_{x_{n+1} y_{n+1}} \in Z_{n}(X, R) \subseteq J$. But $f(x, y) e_{x_{n+1} y_{n+1}}=e_{x_{n+1} x} f e_{y y_{n+1}} \in K$, so that, in this case too, $J \cap K$ is non-zero. The lemma now follows.

If there is a minimal essential ideal of $I(X, R)$, then the intersection of a collection of essential ideals is still essential. The following computes the intersection of the ideals of the previous lemma. We will denote the minimal essential ideal of a ring $T$, when it exists, by $E(T)$. Similarly, $E_{L}(T)$ denotes the minimal essential left ideal of $T$.

LEMMA 2. Suppose $X$ is a locally finite partially ordered set and $R$ a commutative ring with identity. Let $\phi$ be an essential function. Then

$$
\bigcap_{n=1}^{\infty}\left(A_{\phi}+Z_{n}(X, R)\right)=A_{\phi}
$$

PROOF. Let $f \in \bigcap_{n=1}\left(A_{\phi}+Z_{n}(X, R)\right)$ and suppose $f(x, y) \neq 0$. Let $m=|[x, y]|$. Since $f \in\left(A_{\phi}+Z_{m}(X, R)\right)$, we can find $g \in A_{\phi}$ and $h \in Z_{m}(X, R)$ with $f=g+h$. As $h(x, y)=0$ we have that $g(x, y)=f(x, y)$. Hence $[x, y]$ is a maximal interval and $f(x, y) \in \phi([x, y])$. It follows that $f \in A_{\phi}$.

The next lemma tells us when $A_{\phi}$ is essential.

LEMMA 3. Suppose $X$ is a locally finite partially ordered set and $R$ a commutative ring with identity. Let $\phi$ be an essential function. Then $A_{\phi}$ is an essential ideal if and only if each interval of $X$ is contained in a maximal interval. In particular, if one $A_{\phi}$ is essential, they all are. 
ProOF. Assume each interval of $X$ is contained in a maximal interval. Let $K$ be a non-zero ideal of $I(X, R)$. To show that $A_{\phi}$ is essential we check that $K \cap A_{\phi} \neq\{0\}$. Let $f$ be a non-zero element of $K$ and let $x, y \in X$ be such that $f(x, y) \neq 0$. Further, let $[u, v]$ be a maximal interval of $X$ which contains $[x, y]$. Then $e_{u x} f e_{y v}=$ $f(x, y) e_{u v} \in e_{u u} K e_{v v}=\left\{g(u, v) e_{u v} \mid g \in K\right\}$. Further, $e_{u u} K e_{v v}$ is an ideal of $I(X, R)$ contained in $K$. Let $B=\{g(u, v) \mid g \in K\}$. Then $B$ is a non-zero ideal of $R$ having a non-zero intersection with the essential ideal $\phi([u, v])$. Since $\{0\} \neq\left\{r e_{u v} \mid r \in(\phi([u, v]) \cap B)\right\} \subset\left(A_{\phi} \cap K\right)$, we have that $A_{\phi}$ is essential.

Conversely, suppose $A_{\phi}$ is essential and, looking for a contradiction, there exists an interval, $I_{0}=\left[x_{0}, y_{0}\right]$, in $X$, which is not contained in a maximal interval. Let $K$ be the ideal of $I(X, R)$ generated by $e_{x_{0} y_{0}}$. As $A_{\phi}$ is essential, we can find $0 \neq h \in K \cap A_{\phi}$, and thus a maximal interval, $[u, v]$, with $h(u, v) \neq 0$. Since $K$ is generated by $\left\{f e_{x_{0} y_{0}} g \mid f, g \in I(X, R)\right\}$, we must have an $f_{1}, g_{1} \in I(X, R)$ with $\left(f_{1} e_{x_{0} y_{0}} g_{1}\right)(u, v) \neq 0$. But $\left(f_{1} e_{x_{0} y_{0}} g_{1}\right)(u, v)=f_{1}\left(u, x_{0}\right) g_{1}\left(y_{0}, v\right)$ and, if this is to be non-zero, $\left[x_{0}, y_{0}\right] \subseteq[u, v]$. This is a contradiction as it says that $\left[x_{0}, y_{0}\right]$ is contained in a maximal interval. The lemma is then established.

We now give a criterion for an incidence algebra to have a minimal essential ideal. For notational convenience, when $f \in I(X, R)$ and $A$ is a subset of $R$, write Af $=\{$ af $\mid a \in A\}$.

THEOREM 1. Let $X$ be a locally finite partially ordered set and $R$ a commutative ring with identity. Then $I(X, R)$ has a minimal essential ideal, $E(I(X, R))$, if and only if $R$ has a minimal essential ideal, $E(R)$, and each interval of $X$ is contained in a maximal interval. If $E(I(X, R))$ exists, then

$$
\left.E(I(X, R))=\left\langle E(R) e_{u v}\right|[u, v] \text { a maximal interval }\right\rangle .
$$

Proof. Suppose $E(I(X, R))$ exists. Let $\phi$ be an essential function for $X$. From Lemma 1 and Lemma $2, A_{\phi}$ is essential and thus, by Lemma 3, each interval of $X$ is contained in a maximal interval. We now check that $R$ has a minimal essential ideal. To do this it is sufficient to show that the intersection of any class of essential ideals of $R$ is again essential. Let $\left\{K_{i} \mid i \in I\right\}$ be a class of essential ideals of $R$. Here $I$ is an index set. Further, let $[u, v]$ be a maximal interval in $X$, and $\phi_{i}$ the essential function given by $\phi_{i}([u, v])=K_{i}$ and $\phi_{i}([x, y])=R$ for any other maximal interval, $[x, y]$ of $X$. From Lemma $3, A_{\phi_{i}}$ is essential in $I(X, R)$, and since $E(I(X, R))$ exists, $\bigcap_{i \in I} A_{\phi_{i}}$ is essential. But it is easy to see that

$$
\bigcap_{i \in I} A_{\phi_{i}}=\left\{f \in I(X, R) \mid f(u, v) \in \bigcap_{i \in I} K_{i} \text { and } f(x, y)=0 \text { if }[x, y] \text { not maximal }\right\} .
$$

Suppose $B=\bigcap_{i \in I} K_{i}$ is not essential. Then there is a non-zero ideal, $C$, of $R$, such that $C \cap B=\{0\}$. Let $L=\left\{c e_{u v} \mid c \in C\right\}$. Then $L$ is a non-zero ideal of $I(X, R)$ and, 
as $\bigcap_{i \in I} A_{\phi_{i}}$ is essential, there is a non-zero $f \in\left(L \cap\left(\bigcap_{i \in I} A_{\phi_{i}}\right)\right)$. Simultaneously we must have $f=c e_{u v}$, with $c \in C$, and $f=b e_{u v}$, with $b \in B$. This is not possible as $B \cap C=\{0\}$. We conclude that $B$ is essential, and $R$ contains a minimal essential ideal.

Conversely, suppose that $E(R)$ exists and that each interval of $X$ is contained in a maximal interval. Let $D=\left\langle E(R) e_{u v}\right|[u, v]$ maximal $\rangle$. Note that $D=\bigoplus E(R) e_{u v}$, the sum ranging over all maximal intervals $[u, v]$ in $X$. We first check that $D$ is essential. Let $K$ be a non-zero ideal of $I(X, R)$ and $f$ a non-zero element of $K$. Let $x, y \in X$ be such that $f(x, y) \neq 0$ and $[u, v]$ a maximal interval containing $[x, y]$. Then $e_{u x} f e_{y v}=f(x, y) e_{u v}$ and the non-zero ideal $e_{u u} K e_{v v}=\left\{g(u, v) e_{u v} \mid\right.$ $g \in K\} \subseteq K$. Since $E(R)$ is essential, $E(R) \cap\{g(u, v) \mid g \in K\} \neq\{0\}$, and so $E(R) e_{u v} \cap e_{u u} K e_{v v} \neq\{0\}$. Hence $D$ is essential.

To complete the proof we need only check that $D$ is the minimal essential ideal of $I(X, R)$. Let $M$ be an essential ideal of $I(X, R), C$ a non-zero ideal of $R$, and $[u, v]$ a maximal interval in $X$. Then $K_{C}=\left\{c e_{u v} \mid c \in C\right\}$ is a non-zero ideal of $I(X, R)$, and so $K_{C} \cap e_{u u} M e_{v v} \neq\{0\}$. As $e_{u u} M e_{v v}=\left\{m(u, v) e_{u v} \mid m \in M\right\}$, then $L=\{m(u, v) \mid m \in M\}$ is an ideal of $R$ which has a non-zero intersection with $C$. Since $C$ is an arbitrary ideal of $R, L$ is essential. Hence $E(R) \subseteq L$. Therefore, $E(R) e_{u v} \subseteq M$ and $D \subseteq M$.

A point, $x \in X$, is isolated if the connected component of $x$, in its Hasse diagram, is $\{x\}$. The following corollary shows that $E(I(X, R))$ is often nilpotent.

COROLLARY 1. Let $X$ be a locally finite partially ordered set and $R$ a commutative ring with identity. If $E(I(X, R))$ exists then

$$
(E(I(X, R)))^{2}=\bigoplus_{x \text { isolased }}(E(R))^{2} e_{x x} .
$$

In particular, if $X$ has no isolated points, $(E(I(X, R)))^{2}=\{0\}$.

Green and Van Wyk [2] considered when the minimal essential ideal of a structural matrix ring equals the maximal small ideal. The maximal small ideal is the BrownMcCoy radical [3], which, in the incidence ring case under discussion, coincides with the Jacobson radical [5]. If $J(T)$ denotes the Jacobson radical of the ring $T$, then $J(I(X, R)=\{f \in I(X, R) \mid f(x, x) \in J(R)$ for $x \in X\}$ (see [1]). As we have a description of both $E(I(X, R))$ (when it exists) and $J(I(X, R))$, the following result is easily verified. Recall first that a partially ordered set $X$ is of bound $n$, if the longest chain of distinct elements of $X$ is $n$.

THEOREM 2. Let $X$ be a locally finite partially ordered set and $R$ a commutative ring with identity. Suppose $I(X, R)$ has a minimal essential ideal. Then $E(I(X, R))=$ $J(I(X, R))$ if and only if one of the following holds: 
(i) $X$ is a finite antichain, and $E(R)=J(R)$;

(ii) $X$ has no isolated points, $J(R)=\{0\}, E(R)=R$, and $X$ is a finite partially ordered set of bound 2.

Proof. Suppose $E(I(X, R))=J(I(X, R))$. If $f \in E(I(X, R))$, from Theorem 1 it follows that $f(u, v)=0$ for all but a finite number of $[u, v]$. Thus $X$ is a finite partially ordered set. Assume first that $J(R) \neq\{0\}$. Let $x \in X$. Then $J(R) e_{x x} \subseteq$ $J(I(X, R))$ and so, by Theorem $1,[x, x]$ is a maximal interval and $J(R) \subseteq E(R)$. It then follows that $E(R) e_{x x} \subseteq E(I(X, R))$ and $E(R)=J(R)$. Assume, now, that $J(R)=\{0\}$. Then $E(R) e_{x x} \cap J(I(X, R))=\{0\}$ and thus $x$ is not an isolated point. If $y \in X$ is such that $x<y$ then $e_{x y} \in J(I(X, R))$ guarantees that $[x, y]$ is a maximal interval, and that $1 \in E(R)$. It follows that $X$ is of bound 2 and $E(R)=R$. The converse of the theorem is straightforward.

In the following we briefly describe when $I(X, R)$ has a minimal essential left ideal within the lattice of left ideals of $I(X, R)$. The left ideal $A$ of the ring $T$ is an essential left ideal if $A \cap B \neq\{0\}$ for any non-zero left ideal $B$ of $T$. If $M$ is a left $T$-module, then the submodule $N$ is an essential left submodule of $M$ if $N \cap V \neq\{0\}$, for each non-zero submodule $V$ of $M$.

As before, $X$ denotes a locally finite partially ordered set and $R$ a commutative ring with identity. Let $\operatorname{Min}(X)$ be the collection of all minimal elements of $X$ and $\operatorname{Max}(X)$ the collection of all maximal elements of $X$. Of course, $\operatorname{Min}(X)$ and $\operatorname{Max}(X)$ are antichains of $X$, and each interval of $X$ is contained in a maximal interval if and only if $\operatorname{Min}(X)$ and $\operatorname{Max}(X)$ are each maximal antichains.

Let $L=\{f \in I(X, R) \mid f(x, y)=0$ if $x \notin \operatorname{Min}(X)\}$ and $Z_{n}(X, R)$ the ideal defined before Lemma 1 . It is easy to check that $L$ is a left ideal and, for $n$ a positive integer, that $L+Z_{n}(X, R)$ is again a left ideal. Suppose $K$ is a non-zero left ideal of $I(X, R)$, and $0 \neq f \in K$. Further, suppose $f(u, v) \neq 0$, for some $u, v \in X$, with $u$ related to an element, $x \in \operatorname{Min}(X)$. Then $0 \neq e_{x u} f \in(K \cap L)$. If no such $u$ exists, by an argument similar to that in Lemma 1 , we obtain that $f \in\left(Z_{n}(X, R) \cap K\right)$. This shows that $L+Z_{n}(X, R)$ is an essential left ideal of $I(X, R)$. Further, by an argument parallel to that in Lemma 2, we obtain that the intersection of the left ideals $L+Z_{n}(X, R)$, as $n$ ranges over the positive integers, is $L$. We summarize our observations in the following lemma.

LEMMA 4. Suppose $X$ is a locally finite partially ordered set, $R$ a commutative ring with identity, and $n$ a positive integer. Then $L+Z_{n}(X, R)$ is an essential left ideal of $I(X, R)$. Further,

$$
\bigcap_{n=1}^{\infty}\left(L+Z_{n}(X, R)\right)=L .
$$


We now observe when $L$ is an essential left ideal.

LEMMA 5. Suppose $X$ is a locally finite partially ordered set and $R$ a commutative ring with identity. Then $L$ is an essential left ideal of $I(X, R)$ if and only if $\operatorname{Min}(X)$ is a maximal antichain.

Proof. Assume $\operatorname{Min}(X)$ is a maximal antichain. By the remarks preceding Lemma 4 , if $f$ is a non-zero element of a non-zero left ideal, $K$, of $I(X, R)$ and $f(u, v) \neq 0$, then $e_{x u} f \in(K \cap L)$, for any minimal element $x \leq u$ of $X$. This shows that $L$ is an essential left ideal.

Conversely, suppose there exists an $x_{0} \in X$ incompatible with all elements of $\operatorname{Min}(X)$. Then it is easy to check that the left ideal generated by $e_{x_{0} x_{0}}$ does not have any non-zero elements in common with $L$.

We need some additional terminology before presenting a criterion for the existence of a minimal essential left ideal of $I(X, R)$. If $M={ }_{R} M$ is a left $R$-module, the submodule $T$ of $M$ is essential if $T \cap N \neq\{0\}$, for any non-zero submodule $N$ of $M$. We say that ${ }_{R} M$ has a minimal essential submodule, $E_{L}(M)$, if the intersection of all its essential submodules is essential. In order that $I(X, R)$ have a minimal essential ideal, we observed in Theorem 1 that $R$ must have a minimal essential ideal, $E(R)$. This, of course, is equivalent to saying that $R$, as a left $R$-module, has a minimal essential submodule, and it is this latter formulation which leads to a necessary condition for $I(X, R)$ to have a minimal essential left ideal.

Let $\kappa$ be a cardinal number and let $\Pi_{\kappa} R$ denote the product of $\kappa$ copies of the commutative ring $R$. We consider ${ }_{R} \Pi_{\kappa} R$, that is, $\Pi_{\kappa} R$ regarded as a left $R$-module. If ${ }_{R} A$ and ${ }_{R} B$ are left $R$-modules then $A \oplus B$ has a minimal essential submodule, if and only if $A$ and $B$ each do, and $E_{L}(A \oplus B)=E_{L}(A) \oplus E_{L}(B)$. In particular, if $\kappa_{1} \leq \kappa_{2}$ are cardinal numbers then the existence of $E_{L}\left({ }_{R} \Pi_{\kappa_{2}} R\right)$ guarantees the existence of $E_{L}\left({ }_{R} \Pi_{\kappa_{1}} R\right)$. Further, it is easy to check that if $E_{L}\left({ }_{R} \Pi_{\kappa} R\right)$ exists then $R$ has a minimal essential ideal, $E(R)$, and

$$
{ }_{R} \oplus_{\kappa} E(R) \subseteq E_{L}\left({ }_{R} \Pi_{\kappa} R\right) \subseteq{ }_{R} \Pi_{\kappa} E(R)
$$

Let $x \in \operatorname{Min}(X)$ and let $\kappa_{x}=|\{y \in X \mid x \leq y\}|$. We call $R \operatorname{Min}(X)$ essential if $E_{L}\left({ }_{R} \Pi_{\kappa_{x}} R\right)$ exists, for each $x \in X$. We can now describe when $I(X, R)$ has a minimal essential left ideal.

THEOREM 3. Let $X$ be a locally finite partially ordered set and $R$ a commutative ring with identity. Then $I(X, R)$ has a minimal essential left ideal, $E_{L}(I(X, R))$, if and only if $\operatorname{Min}(X)$ is a maximal antichain and $R$ is $\operatorname{Min}(X)$ essential. 
PROOF. Suppose that $I(X, R)$ has a minimal essential left ideal $E_{L}(I(X, R))$. Since the ideal $L$, of Lemma 4 , is the intersection of essential left ideals, it must be essential. Lemma 5 then tells us that $\operatorname{Min}(X)$ is a maximal antichain. We now check that $R$ is $\operatorname{Min}(X)$ essential. Let $x \in \operatorname{Min}(X), S(x)=\{y \in X \mid x \leq y\}$ and $\kappa_{x}=|S(x)|$. It is sufficient to see that ${ }_{R} \Pi_{\kappa_{x}} R$ has a minimal essential submodule. Let $J_{x}$ be an index set of cardinality $\kappa_{x}$ and $\phi_{x}: J_{x} \rightarrow S(x)$ a bijective mapping. Call $f \in I(X, R)$ an $S(x)$ function if $f(u, v)=0$ for $u \neq x$. If $f$ is an $S(x)$ function and $g \in I(X, R)$, then, for $y \in X, g f(x, y)=g(x, x) f(x, y)$. It follows that the left ideal generated by $f$ agrees with the left $R$-module generated by $f$. For each $j \in J_{x}$, let $R_{j}=R$ and $T(x)={ }_{R} \prod_{j \in J_{x}} R_{j}$. We regard $T(x)$ as the collection of functions, $g$, from $J_{x}$ to $R$ with $g(j) \in R_{j}$. If $f$ is an $S(x)$ function, let $\alpha_{x}(f) \in T(x)$ be the element defined by $\alpha_{x}(f)(j)=f\left(x, \phi_{x}(j)\right)$. It is easy to see that $\alpha_{x}$ is a bijective $R$-module mapping from the $R$-submodule of $S(x)$ functions to $T(x)$. Further, if $\alpha_{x}(f)=t$, then the cyclic $R$-submodule of $T(x)$ generated by $t$ corresponds, under $\alpha_{x}^{-1}$, with the cyclic $R$-submodule generated by $f$, which, in turn, agrees with the left ideal of $I(X, R)$ generated by $f$. Let $V=e_{x x} E_{L}(I(X, R))$. Then $V$ is the collection of all $S(x)$ functions in $E_{L}\left(I(X, R)\right.$. Let $\alpha_{x}(V)=\left\{\alpha_{x}(f) \mid f \in V\right\}$. A straightforward verification shows that $\alpha_{x}(V)$ is an $R$-submodule of $T(x)$. If $t_{1}$ is a non-zero element of $T(x)$, then $\alpha_{x}^{-1}(t)$ is an $S(x)$ function and thus the left ideal of $I(X, R)$ that it generates has a non-zero intersection with $E_{L}(I(X, R)$. It follows that there is an $r \in R$ with $r \alpha_{x}^{-1}(t)$ a non-zero element in $V$. Hence $0 \neq r t \in \alpha_{x}(V)$ and $\alpha_{x}(V)$ is an essential submodule of $T(x)$. We check that it is the minimal essential $R$-submodule of $T(x)$. Suppose $U$ is an essential submodule of $T$. For $y \in \operatorname{Min}(X)$, with $y \neq x$, let $W(y)$ be the collection of all $S(y)$ functions contained in $E_{L}(I(X, R)$. Further, let $K$ be the left ideal generated by

$$
\left(\bigcup_{\substack{y \in \operatorname{Min}(x) \\ y \neq x}} W(y)\right) \bigcup \alpha_{x}^{-1}(U) .
$$

Notice that the collection of all $S(x)$ functions in $K$ coincides with $\alpha_{x}^{-1}(U)$. We check that $K$ is an essential left ideal of $I(X, R)$. Then it follows that $E_{L}(I(X, R) \subseteq K$ and so $V \subseteq \alpha_{x}^{-1}(U)$. Hence $\alpha_{x}(V) \subseteq U$ and $\alpha_{x}(V)$ is the minimal essential $R$-submodule of $T(x)$.

Let $f$ be a non-zero function of $I(X, R)$ and $f(u, v) \neq 0$, for $u, v \in X$. There is a $y \in \operatorname{Min}(X)$ with $y \leq u$. Suppose, first, that $y \neq x$. The left ideal generated by $e_{y u} f(u, v)$ has a non-zero intersection with $E_{L}(I(X, R))$, and so there is a non-zero $S(y)$ function, $g$, common to these two ideals. Since $g \in W(y)$, then $g \in K$. Suppose now that $y=x$. Then $e_{x u} f$ is a non-zero $S(x)$ function and thus $t_{1}=\alpha_{x}\left(e_{x u} f\right)$ is a non-zero element of $T(x)$. Since $U$ is an essential module, there is an $r_{1} \in R$ with $r_{1} \alpha_{x}\left(e_{x u} f\right)$ a non-zero element of $U$. But $0 \neq r_{1} e_{x u} f \in \alpha_{x}^{-1}(U) \subseteq K$ and, thus, in 
either case, the left ideal generated by $f$ has a non-zero intersection with $K$. It follows that $K$ is essential and that $R$ is $\operatorname{Min}(X)$ essential.

Conversely, suppose that $R$ is $\operatorname{Min}(X)$ essential and that $\operatorname{Min}(X)$ is a maximal antichain. Let $x \in \operatorname{Min}(X)$. Let $P_{x}$ be the essential submodule of the product of $\kappa_{x}$ copies of $R$ regarded as a left $R$-module. Then $\alpha_{x}^{-1}\left(P_{x}\right)$ is a left ideal of $I(X, R)$ consisting of $S(x)$ functions. Let

$$
E_{L}(I(X, R))=\bigoplus_{x \in \operatorname{Min}(X)} \alpha_{x}^{-1}\left(P_{x}\right) .
$$

Using similar methods to the previous part, it is straightforward to check that $E_{L}(I(X, R))$ is the minimal essential left ideal of $I(X, R)$.

The following consequence of the previous theorem and its proof gives a description of the minimal essential left ideal in a special situation.

COROLLARY 2. Suppose $R$ is a commutative ring with identity and $X$ a locally finite partially ordered set having the property that, for $x \in X,|\{y \in X \mid x \leq y\}|<\infty$. Then $I(X, R)$ has a minimal essential left ideal, $E_{L}(I(X, R))$, if and only if $\operatorname{Min}(X)$ is a maximal antichain and $R$ has a minimal essential ideal, $E(R)$.

Suppose $E_{L}(I(X, R))$ exists. For $x \in \operatorname{Min}(X)$, let

$$
A(x)=\{f \in I(X, R) \mid f(x, v) \in E(R), f(u, v)=0 \text { otherwise }\} .
$$

Then $A(x)$ is a left ideal of $I(X, R)$ and

$$
E_{L}(I(X, R))=\bigoplus_{x \in \operatorname{Min}(X)} A(x)
$$

\section{References}

[1] P. Doubilet, G.-C. Rota and R. P. Stanley, 'On the foundation of combinatorial theory VI: The idea of generating function', in: Finite operator calculus (Academic Press, New York, 1975) pp. 267-318.

[2] B. W. Green and L. Van Wyk, 'On the small and essential ideals in certain classes of rings', $J$. Austral. Math. Soc. (Series A) 46 (1989), 262-271.

[3] N. V. Loi and R. Wiegandt, 'Small ideals and the Brown-McCoy radical', Radical theory (Eger 1982), Colloq. Math. Soc. János Bolyai 38 (North Holland, Amsterdam, 1985).

[4] L. Rowen, Ring theory, vol. I (Academic Press, Boston, 1988).

[5] E. Spiegel and C. J. O'Donnell, Incidence algebras (Marcel Dekker, New York, 1977).

Department of Mathematics

University of Connecticut

Storrs, CT 06269

USA

e-mail: spiegel@math.uconn.edu 